Villous adenomas are common lesions of the gastrointestinal tract but they are rarely located in the urinary tract including the urinary bladder. There are a few case reports and series in the literature. Here we report a 43-year-old male patient who had a polypoid lesion located on the left lateral wall of the urinary bladder. Transurethral resection was performed. The diagnosis was 'villous adenoma of urinary bladder' with clinical and histopathological findings.

Villous adenomas are mostly seen in elderly males. The coexistence of villous adenoma with adenocarcinoma, squamous cell carcinoma, and urothelial carcinoma was observed but there is not clear evidence about progression to carcinoma, in spite of its colonic counterpart. Generally complete surgical resection is accepted as curative but there are no exact data about followup and recurrence. By presenting this case, we aim to emphasize that it is a rare but important lesion because of coexistence with malignancies and uncertain malignant potential.

Key words: villous adenoma, location, urinary, bladder.

\section{Villous adenoma of the urinary bladder: rare location}

\author{
Esin Atik ${ }^{1}$, Bülent Akansu', Mürsel Davarci ${ }^{3}$, Mehmet Inci ${ }^{3}$, \\ Fatih Yalcinkaya ${ }^{3}$, Murat Rifaioglu ${ }^{3}$
}

1Department of Pathology, Mustafa Kemal University Medical Faculty, Hatay, Turkey 2Division of Pathology, Diyarbakir State Hospital, Diyarbakir, Turkey ${ }^{3}$ Department of Urology, Mustafa Kemal University Medical Faculty, Hatay, Turkey

\section{Introduction}

Villous adenomas are common lesions of the gastrointestinal tract but they are rarely located in the urinary tract including the bladder. There are a few case reports and series in the literature. They should be accepted as important lesions because the malignancy potential of them is still not known $[1,2]$. Here we report an additional case to draw attention to this important but rare lesion.

\section{Case report}

A 43-year-old male patient was admitted to our hospital with the complaint of non-coagulated hematuria and pain. He indicated that he had hematuria several times before in his medical history. There was no remarkable finding in physical examination. A polypoid lesion $14 \mathrm{~mm} \times 10 \mathrm{~mm}$ in diameter located on the left lateral wall of the bladder was detected in ultrasonography. Transurethral resection was performed by the urology department and material was sent to the pathology department for histopathological evaluation. The sections prepared with hematoxylin and eosin showed that the lesion was composed of villous structures lined by intestinal epithelium with mild dysplastic changes in some nuclei and abundant mucinous substance, which were remarkable microscopically (Fig. 1). The lesion was stained diffusely positive with carcinoembryonic antigen (CEA), focal positive with cytokeratin 20 (CK-20), cytokeratin 7 (CK-7) and negative with epithelial membrane antigen (EMA). The diagnosis was 'villous adenoma of urinary bladder' with these clinical and histopathological findings. A conclusion about the possibility of having a malignancy potential in these lesions was also included in the pathology report and close follow-up of the patient was suggested to the clinic.

\section{Discussion}

Although villous adenomas are commonly seen in the gastrointestinal tract, they are rare in the urinary tract including the urinary bladder [1]. There are a few case reports and series in the English literature.

Villous adenomas are mostly seen in elderly males. The mean is 57 years of age with the range from 33 to 79 [1]. The patients generally complain about hematuria, irritative symptoms and rarely mucusuria [1-5]. Bladder dome and the posterior wall are the most frequent sites. It was located on the lateral wall in our case.

The embryological origin of distal colorectal and urinary bladder is closely related. They develop from the partitioning of the cloaca by the urorectal septum [1,3]. It is postulated that remnants of cloaca may exist in the bladder and they can cause a glandular epithelial neoplasm. There is also a suggestion that these neoplasms originate from injured stem cells of urothelium which result in glandular metaplasia [3]. Chronic irritation such as infection, 


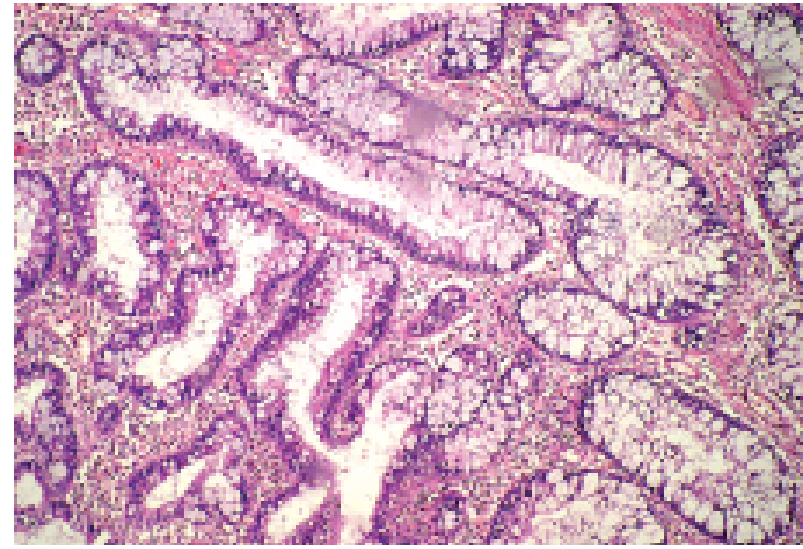

Fig. 1. Villous structures lined by intestinal epithelium with mild dysplastic changes in some nuclei (HE, magnification 100x)

chemical injury or presence of calculi may result in intestinal metaplasia of epithelium $[2,4,6]$.

Not surprisingly, villous adenomas are similar to gastrointestinal ones, histologically. They both consist of villous structures lined by columnar epithelial cells with some goblet type mucin producing cells. Some epithelial cells may exhibit nuclear atypia and dysplastic changes [1]. Villous adenomas of the urinary tract also have similar immunohistochemical properties with their colonic counterpart. They stain positive with carcinoembryonic antigen (CEA) and cytokeratin 20 (CK-20), but generally negative with epithelial membrane antigen (EMA) [1]. Cytokeratin 7 (CK-7) is positive in approximately $50 \%$ of urinary tract lesions in contrast to intestinal ones [1]. Consistently with the literature, our sections stained strongly and diffusely positive with CEA (Fig. 2), focal positive with CK-2O and CK-7, and negative with EMA. Neutral acid and sialomucins secreted by adenomas can be shown with periodic acid-Schiff and alcian blue stains as well [4].

The epitope for mAbDas1 which was firstly identified on normal colonic epithelium was used in one study, and found positive in 8 of 10 cases. This epitope was also positive in neoplastic epithelium of the colon, but negative in normal, inflamed and neoplastic urothelium. It was expressed in urinary tract lesions exhibiting glandular differentiation. Molecular studies showed DNA aneuploidy and increased expression of p53 in villous adenomas [3].

Cystitis glandularis, in situ and well-differentiated adenocarcinoma should be considered for differential diagnosis. There are no well-formed villous structures which were characteristic for villous adenomas in cystitis glandularis. Dysplastic appearance in pseudo-stratified epithelium with enlarged, crowded and hyperchromatic nuclei is remarkable in a well-differentiated adenocarcinoma [1]. Sometimes invaginations of adenomatous epithelium into the stroma can cause difficulties for differential diagnosis from invasive neoplasm $[3,5]$.

The coexistence of villous adenoma with in situ or invasive adenocarcinoma, squamous cell carcinoma, and urothelial carcinoma was shown in some studies [3, 4]. On the other hand, there is no clear evidence about progression to carcinoma, in spite of its colonic counterpart [1].

Since it is a rare lesion, there are no exact data about fol low-up and recurrence. It was reported that no evidence about

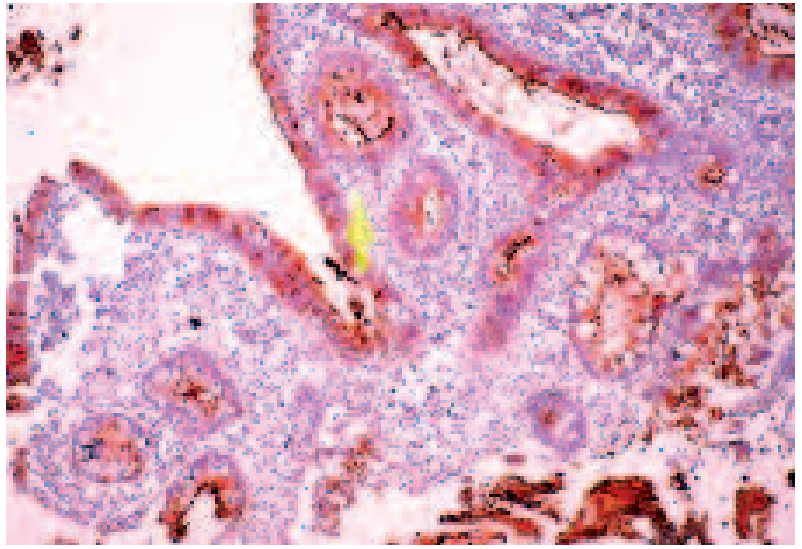

Fig. 2. Carcinoembryonic antigen (CEA) was stained positive (CEA, magnification 100x)

recurrence was found in patients with pure villous adenoma in some studies $[3,4]$. However, it is uncertain whether an untreated lesion may progress to invasive neoplasm [1, 4].

Generally complete surgical resection is accepted as curative and prognosis is excellent in patients with isolated villous adenoma [4]. But recurrence or distant metastasis may be seen in patients with coexistent malignancy, and more aggressive treatment can be indicated [3, 4].

In conclusion, villous adenomas are important lesions because of coexistence with malignant neoplasms and uncertain malignant potential. So the clinician and the pathologist should be aware of this situation, and any urinary tract lesion diagnosed as villous adenoma of the urinary tract must be thoroughly sampled.

\section{References}

1. Sung W, Park BD, Lee S, Chang SG. Villous adenoma of the urinary bladder. Int J Urol 2008; 15: 551-3.

2. Chaudhuri A, Sandhu DP, Xuereb J. Villous adenoma of the urinary bladder. BJU Int 1999; 84: 177-8.

3. Seibel JL, Prasad S, Weiss RE, Bancila E, Epstein JI. Villous adenoma of the urinary tract: a lesion frequently associated with malignancy. Hum Pathol 2002; 33: 236-41.

4. Cheng L, Montironi R, Bostwick DG. Villous adenoma of the urinary tract: a report of 23 cases, including 8 with coexistent adenocarcinoma. Am J Surg Pathol 1999; 23: 764-71.

5. Adegboyega PA, Adesokan A. Tubulovillous adenoma of the urinary bladder. Mod Pathol 1999; 12: 735-8.

6. Corica FA, Husmann DA, Churchill BM, Young RH, Pacelli A, Lopez-Beltran A, Bostwick DG. Intestinal metaplasia is not a strong risk factor for bladder cancer: study of 53 cases with long-term follow-up. Urology 1997; 50: 427-31.

\section{Address for correspondence}

Mürsel Davarci MD, Assist Prof.

Department of Urology, Faculty of Medicine,

Mustafa Kemal University

31100 Hatay, Turkey

tel. $+90-3262291000$ (Ext. 2917)

fax $+90-3262455305$

e-mail: mdavarci1970@hotmail.com

Submitted: $\quad$ 7.04.2011

Accepted: $\quad$ 18.01.2012 